\title{
Detonation nanodiamonds: new aspects in the theory and practice of synthesis, properties and applications
}

\author{
Valery Yu. Dolmatov, ${ }^{\mathrm{a} *}$ Alexander N. Ozerin, ${ }^{\mathrm{b}}$ Inna I. Kulakova, ${ }^{\mathrm{c}}$ Oleksandr O. Bochechka, ${ }^{\mathrm{d}}$ \\ Natalia M. Lapchuk, ${ }^{\mathrm{e}}$ Vesa Myllymäki, ${ }^{\mathrm{f}}$ Asko Vehanen ${ }^{\mathrm{f}}$
}

\author{
a Federal State Unitary Enterprise, Special Design and Technology Bureau 'Tekhnolog', \\ Sovetsky prosp. 33a, 192076 Saint Petersburg, Russian Federation \\ ${ }^{b}$ N.S.Enikolopov Institute of Synthetic Polymer Materials, Russian Academy of Sciences, \\ ul. Profsoyuznaya 70, 117393 Moscow, Russian Federation \\ ${ }^{c}$ Department of Chemistry, Lomonosov Moscow State University, \\ Leninskie Gory 1, stroenie 3, 119991 Moscow, Russian Federation \\ ${ }^{d}$ V.N.Bakul Institute of Superhard Materials of the National Academy of Sciences of Ukraine, \\ ul. Avtozavodskaya 2, 04074 Kiev, Ukraine \\ ${ }^{e}$ Faculty of Physics, Belarusian State University, \\ ul. Bobruiskaya 5, 220030 Minsk, Belarus \\ ${ }^{f}$ Carbodeon Ltd. Oy, \\ Pakkalankuja 5, 01510 Vantaa, Finland
}

\begin{abstract}
The review describes the current state of research concerning detonation nanodiamonds, including production, methods of studying the properties and applications. The main achievements in the theory and practice of synthesis of detonation nanodiamonds over the past 15 years are addressed systematically; the influence of control factors on this process, performed using single or mixed explosives, are discussed. A new highly economical and environmentally friendly method for chemical purification of nanodiamonds is described. The operational characteristics of new materials based on nanodiamonds are presented. The application prospects of nanodiamonds in traditional and new fields are demonstrated.

The bibliography includes 214 references.
\end{abstract}

\footnotetext{
V.Yu.Dolmatov. Doctor of Technical Sciences, Head of the Research Laboratory of the SDTB Tekhnolog.

E-mail: diamondcentre@mail.ru

Current research interests: theoretical and applied principles of synthesis and chemical purification of detonation nanodiamonds, development of new methods for producing modified and doped nanodiamonds, surface chemistry of nanomaterials, development of techniques for using nanodiamonds in various fields of technology.

A.N.Ozerin. Doctor of Chemical Sciences, Scientific Supervisor of ISPM RAS.

E-mail: ozerin@ispm.ru

Current research interests: high-molecular-weight compounds; physics and mechanics of polymers and polymer composite materials; chemistry of macromolecular compounds; technology for processing polymers and polymer composites; condensed matter physics; chemical physics; X-ray diffraction analysis of polymers; computer simulation.

I.I.Kulakova. PhD in Chemistry, Leading Researcher at the Division of Petroleum Chemistry and Organic Catalysis, Department of Chemistry, MSU.

E-mail: inna-kulakova@yandex.ru

Current research interests: heterogeneous catalysis, surface chemistry of solids, chemical modification of detonation nanodiamonds, application of modified nanodiamonds in catalysis and biomedicine.

O.O.Bochechka. Doctor of Technical Sciences, Deputy Director for Research, ISM NAS of Ukraine.
}

E-mail: bochechka@ism.kiev.ua

Current research interests: high-pressure and high-temperature interaction of diamond (carbon) powders with gases $\left(\mathrm{O}_{2}, \mathrm{CO}, \mathrm{CO}_{2}\right)$ and metal melts $(\mathrm{Ni}, \mathrm{Co}, \mathrm{Cu})$; sintering of nanopowders: diamonds, $\mathrm{SiC}$, etc.

N.M.Lapchuk. PhD in Physics and Mathematics, Associate Professor, Department of Physics of Semiconductors and Nanoelectronics, Faculty of Physics, BSU.

E-mail: lapchuk@bsu.by

Current research interests: electron paramagnetic resonance of carboncontaining materials: natural and synthetic diamonds, pyrolytic graphite, polymers, brown and hard coals, crude oil and oil refining products; radiation resistance of silicon and carbon-containing materials.

V.Myllymäki. PhD in Chemistry, Chief Executive Officer,

Carbodeon Ltd. Oy.

E-mail: vesa.myllymaki@carbodeon.com

Current research interests: synthesis of detonation nanodiamonds, modification of their surface, development of their applications in electroplating, polymer chemistry and other fields of technology. A.Vehanen. Doctor of Technology, Director of Carbodeon Ltd. Oy. E-mail: asko.vehanen@carbodeon.com

Current research interests: synthesis of detonation nanodiamonds, modification of their surface, development of their applications in electroplating, polymer chemistry and other fields of technology.

Translation: Z.P.Svitanko 


\section{Contents}

1. Introduction

2. Detonation synthesis of nanodiamonds

2.1. Possible mechanism of the synthesis of nanodiamonds

2.2. Regularities of the detonation synthesis of nanodiamonds

2.3. Diamond blend

2.3.1. Particle morphology of the diamond blend

2.3.2. Isolation of detonation nanodiamonds from the diamond-containing blend

3. Properties of detonation nanodiamonds

3.1. Structure and composition of primary particles

3.2. Stability of suspensions of detonation nanodiamonds

3.3. Optical properties of detonation nanodiamonds

3.3.1. IR spectra

3.3.2. Raman spectra and photoluminescence

3.4. Characteristic features of paramagnetism of doped detonation nanodiamonds

3.4.1. Characteristic features of paramagnetism of DNDs doped with boron and phosphorus atoms during the synthesis

3.4.2. Dispersion of magnetic susceptibility

3.4.3. Characteristic features of formation of highly conductive structures in DND powders under conditions of electron paramagnetic resonance

3.4.4. Thermal stability of detonation nanodiamonds

3.5. Methods for chemical modification of the surface of detonation nanodiamonds

4. Applications of detonation nanodiamonds

4.1. Fluorinated nanodiamonds as unique neutron reflectors

4.2. DND-CNT hybrid material

4.3. Electrochemical metal-diamond coatings

4.3.1. Chromium-diamond coatings

4.3.2. Nickel-diamond coatings

4.3.3. Electrochemical diamond-containing anodized aluminium oxide coatings

4.3.4. Silver-diamond coatings

4.3.5. Gold-diamond coatings

4.3.6. Zinc-diamond coatings

4.4. Bulk metal-nanodiamond composites

4.5. Composite materials based on polymers

4.5.1. Elastomer-diamond composite materials

4.5.2. Polymer-diamond composites based on thermoplastics

4.5.3. Tribology of polymer compositions

4.5.4. Polymer-DND composites for special applications

4.6. Antifriction oil compositions with detonation nanodiamonds

4.7. Catalysts based on detonation nanodiamonds

4.8. Polycrystalline materials made of nanodiamonds

4.9. Modified detonation nanodiamonds for biomedical applications

4.9.1. Nanodiamonds as fluorescent labels

4.9.2. Nanodiamonds for drug delivery

4.9.3. Imaging of nanodiamonds

4.9.4. Specific activity of DND - glycine conjugate in vivo

5. Conclusion

List of abbreviations:

ATR - attenuated total reflection,

BAS - biologically active substance,

BTF - benzotrifuroxan, benzotris(1,2,5-oxadiazole

1,4,7-trioxide),

CEC - composite electrochemical coatings,

CNT - carbon nanotubes,

$\mathrm{CRZ}$ - chemical reaction zone,

DB - diamond detonation blend,

DGEBA - diglycidyl ether of bisphenol A,

DND - detonation nanodiamond,

DP - detonation products,

DS - drug substance,

FL - fluorescence,

HPV - high-pressure vessel,

$\mathrm{NV}$ - nitrogen vacancy,
OB - oxygen balance,

OPBI - highly heat-resistant poly[2,2'-( $p$-oxydiphenylene)-5, $5^{\prime}$-bibenzimidazole],

PA6 - polyamide 6 (kapron),

PDMS - polydimethylsiloxane,

PPase - pyrophosphatase,

PVA - polyvinyl alcohol,

RDX - hexogen (cyclotrimethylenetrinitramine),

TATB - 1,3,5-triamino-2,4,6-trinitrobenzene,

TETA - triethylenetetramine,

TG - a charge of TNT (trotyl) and RDX (hexogen),

TNT - 2,4,6- trinitrotoluene (trotyl),

UHMWPE - ultrahigh molecular weight polyethylene,

UPTFE - ultradispersed polytetrafluorethylene,

VTMOS - vinyltrimethoxysilane. 


\section{Introduction}

More than 50 years have passed since the discovery of detonation nanodiamonds (DNDs), 4-6 $\mathrm{nm}$ carbon particles. The last decade witnessed a revival of the interest in DNDs. The range of raw materials for the synthesis of nanodiamonds is being expanded: new explosives extracted from ammunition are now used. Modified and doped DNDs were obtained, a new environmentally safe and economically feasible processes for their chemical purification appeared. This led to a significant change in the properties of DNDs (for example, high conductivity ${ }^{1}$ arising upon doping) and increased the potential for their use in micro- and nanoelectronics. Studying the properties of DNDs and DND-based materials expanded their applications in traditional and new fields.

Not all important DND characteristics correspond to analogous values of large crystallites: the heat conductivity of the DND powder is two orders of magnitude lower than that of a bulk crystal; the radiation resistance of the crystallite can be high only when there are no metal or nonmetal impurities on the surface or in the pores of the DND powder; chemical inertness of the DND core is only implied, since the functional groups present on the nanocrystal surface make it, conversely, fairly chemically reactive. Doping of DND crystallites with heteroatoms (nitrogen, silicon, phosphorus, boron) results in the change in optical, magnetic and thermophysical properties. Currently, nitrogen doping of DND crystallites to give nitrogen vacancy (NV) centres is considered to be most promising. These nanocrystals can be regarded as elements of a quantum computer. The generation of NV centres in DND crystallites can give an impetus for the development of nanodiamond spintronics.

The studies of properties of pristine and modified DNDs are being expanded, and the number of relevant publications increases every year. ${ }^{2-4}$ The interest is evidently focused on practical application of DNDs in various fields of science, technology, medicine and biology.

Detonation nanodiamonds can serve as examples of diversity and inexhaustibility of the properties of the nanoworld. They belong to the family of nanocarbons, which includes fullerenes, carbon nanotubes, graphene, globular nanocarbon, onions and other structures, and are among the few products that are commercially manufactured. Traditionally, DNDs are produced in industry starting from mixtures of 2,4,6-trinitrotoluene (TNT) and RDX (cyclotrimethylenetrinitramine) in which the component ratio is $50: 50$ or $60: 40$. Other aromatic nitro derivatives, for example, tetryl ( $N$-methyl- $N$-2,4,6-tetranitroaniline), have also been proposed for this purpose. The DND production at the Plant of Plastics (Kopeisk, Russia) is wholly based on the use of tetryl.

The originality of the method of DND synthesis consists in the fact that the 'surplus' carbon atoms in the molecules of explosives serve as the source of carbon, that is, the starting compounds have a negative oxygen balance $(\mathrm{OB}) .^{\dagger}$ In other words, they contain less oxygen than it is necessary for oxidation of all combustible components. The use of

$\dagger$ Oxygen balance is the mass of oxygen that remains after oxidation of all carbon contained in explosive molecule to give carbon dioxide and oxidation of all hydrogen to give water divided by the mass of the taken explosive, expressed in percent. Nitrogen is assumed to remain in the free state.
DNDs in various industry branches is driven by the need to crucially improve the performance characteristics of composite materials. With the existing level of development of the synthesis and understanding of application prospects of DNDs, they are now considered among the most advantageous carbon nanomaterials for nanotechnologies.

The purpose of the present review is to systematize the available data on DNDs, taking into account their unique properties, new production processes and applications in various fields of science and technology. This is not an exhaustive description, in view of the limited size of the review, but an account specially addressing the above aspects.

\section{Detonation synthesis of nanodiamonds}

\subsection{Possible mechanism of the synthesis of nanodiamonds}

The views on the mechanism of the detonation synthesis of DNDs that prevailed a decade ago are described in detail. ${ }^{5-7}$ This description is fragmentary and often contradictory. In view of the enormous temperature $(3000-4300 \mathrm{~K})$ and pressure $(20-30 \mathrm{GPa})$, thousands of chemical reactions involved, and, what is most important, short time of the detonation transformation of explosives $(0.1-0.3 \mu \mathrm{s})$, currently there is no evidence-based proof for the processes that take place in the space from the detonation wave front to the Chapman-Jouguet plane, the chemical reaction zone ${ }^{\S}(\mathrm{CRZ})$. Therefore, it is also difficult to conceive the chemical and physical processes that originate upon expansion of the gaseous detonation products (DP) and involve condensed carbon. It is believed that at least $80 \%$ of the explosion energy is released in the CRZ, while correspondingly $\sim 20 \%$ of the energy is released behind the Chapman - Jouguet plane.

In many studies (e.g., Refs $8-12)$, it is assumed that the major process of formation of DND crystallites starts after the detonation wave front has propagated up to the Chapman-Jouguet plane and ends much further from this plane upon DP expansion. The growth of DNDs is terminated not only because of decrease in the pressure and temperature, but rather due to decrease in the concentration of carbon radicals.

Meanwhile, there are different points of view on the DND formation process. The use of small-angle X-ray scattering made it possible ${ }^{13,14}$ to study the evolution of carbon particle size since the appearance of explosive detonation front. Immediately behind the detonation front, the authors detected condensed carbon nanoparticles with a density exceeding the density of plasma in the CRZ and determined the size of these particles to be up to $2 \mathrm{~nm}$. As the process goes on, the diameter of dense nanocarbon particles $(d)$ increases to reach, within $\sim 4.5 \mu \mathrm{s}$, different values depending on the type of initial explosive. For explosives such as 1,3,5-triamino-2,4,6-trinitrobenzene (TATB), a mixture of TNT and RDX (TG), and benzotrifuroxan (BTF), the $d$ values were as follows

$\$$ Plane in which the energy release stops and the substance flux velocity is equal to the local sound velocity.

$\S$ Chemical reaction zone is the area located immediately behind the detonation front of the explosive (hundreds of micrometres) separating the initial explosives from gaseous explosion products. In this zone, heat evolution takes place, its temperature is $\sim 3000-4500 \mathrm{~K}$, the pressure is $\sim 18-35 \mathrm{GPa}$ and the substance density is $\sim 2.3 \mathrm{~g} \mathrm{~cm}^{-3}$.

- Gaseous and solid products formed upon detonation of the explosive. 